\title{
Torture survivors' symptom load compared to chronic pain and psychiatric in-patients
}

\author{
Uwe Harlacher, PhD*, Linda Nordin**, Peter Polatin, MD, MPH, Clin. Prof.*** \\ psychotherapist, PhD
}

\begin{abstract}
Before their entry into the rehabilitation program at the Rehabilitation and Research Centre for Torture Victims ('RCT') in Copenhagen, the degree of symptoms of a group of resettled traumatized refugees was assessed by means of two rating scales: the Disability Rating Index (DRI) ( $\mathrm{n}=197)$, measuring pain-related functional disability, and the Hospital Anxiety and Depression Scale (HADS) $(n=147)$. The results obtained were compared with other patient populations, which included (1) a large Swedish mixed pain group and (2) various groups of pain patients previously investigated in the validation study of the DRI scale. The DRI scores of the refugee group were comparable to, or higher than, those of the pain groups, except for patients suffering from multiple sclerosis. The degree of anxiety and depression was found to be considerably greater in the refugee group than in the pain groups. Another recently published Danish study comparing traumatized refugees with psychiatric in-patients in
\end{abstract}

\footnotetext{
*) Nunca Mas International Network for Human Rights and Psycho-social Response

$\star \star)$ Lund University, Sweden

$\star \star \star)$ George Washington University, Harvard Program in Refugee Trauma, Boston, MA, USA
}

Correspondence to: uwe.harlacher@hotmail.com terms of Health of Nation Outcome Scores (HoNOS) documented a higher degree of psychiatric disability for refugees. Based on the hypothesis that the observed differences in this study were underestimated due to the exclusion of refugees with psychotic symptoms and substance abuse, a partial re-analysis of the data was carried out by calculating effect sizes with and without the items measuring these symptoms. Controlling for the exclusion of the critical items resulted in a more pronounced difference between the refugees and psychiatric inpatients. Based on the data compared in this study, traumatized refugees are shown to suffer from multiple problems, including chronic pain, at a high symptom-level. This challenges prior clinical assumptions that single factors like PTSD can explain all symptoms.

Keywords: Functional disability, DRI (Disability Rating Index) HADS (Hospital Anxiety and Depression Scale), traumatized refugees, torture survivors

\section{Background}

Since the establishment of a rehabilitation center for torture survivors in Denmark in the early 1980's by the Rehabilitation and Research Center for Torture Survivors (renamed DIGNITY in 2012), similar treatment centers have been established in industrialized and developing countries 
delivering rehabilitation for survivors of torture or similar traumatization. The survivors receiving rehabilitation in industrialized countries have almost exclusively a background as resettled refugees and are referred to in this paper as traumatized refugees (TR), referring to survivors of torture as well as other more or less severe traumatization.

Within the field of TR rehabilitation, there has been a general dearth of empirical research and quantitative documentation. ${ }^{1}$ Most Danish clinicians working with TR have recognized that their patients have high symptom loads, which, due to lack of quantitative data, has been difficult to document and to publish within the medical literature. On review of the published literature relating to treatment of torture survivors, the impression is given that the primary difficulties after being tortured are PTSD and depression since outcome research focuses on these problems, ${ }^{1,2}$ even though other problems are mentioned in elements of the literature. ${ }^{3}$ Most Danish TR therapists have observed multiple problem domains, such as severe sleep disturbance, cognitive deficits, impulse control problems, social dysfunctions (e.g. conflicts with municipalities, poverty, loneliness, and discrimination), and somatic symptoms, particularly chronic pain. Pain as a major problem is often either ignored or interpreted as secondary to PTSD and/or other psychiatric disorders. Whilst it is well known that chronic pain typically develops out of acute pain due to physical injuries, e.g. after surgery or car accidents, physical injuries inflicted by torture are often not recognized as a potential primary cause for the development of chronic pain. ${ }^{4}$

Systematic quantitative data collection and an on-going monitoring system, now present in most TR treatment centers in
Denmark, will soon improve the empirical knowledge base by providing data that can be used to document the problems of TR patients in comparison with other patient groups. Currently some data are already available for comparative purposes.

Between 2006 and the end of 2011, monitoring-data were collected from a proportion of patients before starting treatment at RCT. Since more than $90 \%$ of the RCT-clientele suffer from chronic pain, the Disability Rating Index (DRI), which measures pain-related disability to conduct activities of daily living, was one of the instruments used in the monitoring system. The Disability Rating Index (DRI) is a self-rating instrument published in 1994 for the assessment of disability caused by impairment of common functions. ${ }^{6}$ It is widely used for the measurement of pain-related disability. ${ }^{7}$ It consists of 12 items, addressing reduced functioning in everyday life situations that are answered on a visual analog scale (VAS) ranging between 0 , anchored by "no difficulty", and 100, anchored by "complete difficulty". A summarizing "index" is computed for the average of all 12 items and is referred to throughout this text.

Anxiety and depression are two additional problems frequently encountered among RCT-patients, and were quantitatively measured by the Hospital Anxiety and Depression Scale (HADS). HADS is a commonly used self-rating instrument for the measurement of anxiety and depression, published in $1983 .{ }^{8}$ Both subscales contain seven items each that are answered in categories between 0-3. Higher scores indicate higher symptom levels.

In this study, the pre-treatment DRI and HADS scores are compared with the scores on these same instruments from other patient-groups prior to treatment. It is of interest to compare the symptom loads 
between different patient groups, including groups of chronic pain patients and psychiatric patients, so as to better understand similarities and differences, with improving treatment as the ultimate purpose.

Palic et al. ${ }^{5}$ compared traumatized refugees (including $31.25 \%$ who were torture survivors) at the Clinic for PTSD and Transcultural Psychiatry, Aarhus University Hospital (CPTP) with psychiatric in-patients treated within the same Danish region. In this study, the Health of Nations Outcome Scores (HoNOS) was used to rate psychiatric disability. The HoNOS is an observer-based rating instrument including 12 items which cover a wide range of disabilities. The refugee-sample had a higher degree of psychiatric disability than any of the six psychiatric in-patient subgroups. In the Palic study, refugee-patients suffering from psychotic symptoms and substance abuse were excluded, so that the psychiatric ratings of the refugee-sample were probably underestimated. We have attempted to partially re-analyze the Palic data in order to address this lacuna.

\section{Method}

\section{Patients treated at RCT}

At the relevant time, the treatment at the RCT consisted of multidisciplinary rehabilitation for about a year in three different contexts as follows: individual (approximately $50 \%$ of patients), group based (approximately $25 \%$ ) and family therapy based (approximately $25 \%$ ). The therapeutic goals were the same irrespective of treatment format.

Patients were, with a few exceptions, adults. The family treatment format included children under the age of 18 . However, only data from adult "index patients" are included in the samples described in this text. The primary target group at RCT included both survivors of torture and traumatization similar to torture (e.g. survivors of organized violence). Patients suffering from present psychotic disorder and severe substance abuse were excluded.

Unlike other Danish TR treatment centers, only patients suffering from multiple problems and in need of multidisciplinary rehabilitation were accepted on the RCT program. Patients with a single diagnosis of PTSD or chronic pain were referred to other treatment providers. These other providers had a lower proportion of torture survivors, e.g. about $30 \%$ at CPTP, the center where Palic et al. conducted their study, compared to about $80 \%$ at RCT. Since to be treated at the RCT patients must be recognized as refugees by the state, or have another type of leave to remain, there were no cases of acute traumatization and the traumatization had typically occurred many years ago. The shortest period since traumatization was two years prior to the first contact with RCT. The patients were referred by general practitioners, specialized physicians (mostly psychiatrists) and hospitals, and rehabilitation treatment was part of the public health system and free of charge. The vast majority of adult patients had minimal skills in the Danish language, so that the percentage of use of interpreters for group sessions was $100 \%$, for family therapy sessions around $95 \%$ and for individual sessions about $80 \%$ (see additional information about language in Table 1.).

Both DRI and HADS data were collected before a patient started treatment at RCT. During the period of this study, about 100 patients per year received treatment and some of them were asked to complete the HADS, DRI or both. The HADS was administered by psychologists and the DRI by physiotherapists. In most cases the officially recognized and validated translation of the instruments were used, most commonly Arabic and Farsi.

The DRI data were collected between 
March 2006 and December 2011, and are available for 197 cases, representing approximately $34 \%$ of all patients starting treatment during this period. HADS data are available for 147 cases collected between October 2008 and May 2010, representing approximately $60 \%$ of all patients during this period. For 112 patients, only DRI-data are available and for 62, only HADS-data are available. For 259 patients, either DRI, HADS or both $(n=85)$ are available, representing approximately $44 \%$ of all patients.

Doubtful cases and missing information in the available data were treated as follows. Three DRI cases were registered in the database with all data missing and were excluded. One case with zero scores in all items was included. One HADS-case with missing data in nine items was excluded. For 13 cases one to three (in total 25) missing item scores have been substituted by the mean score of the available items of the affected subscale and the cases have been included. Several organizational and technical shortcomings are responsible for the incomplete data. The data collection initiated by psychologists and physiotherapists was not synchronized during the whole period and the electronic system to store the data was changed during the observation period, which caused a time gap between the termination of the previous system and the introduction of the new one. We are however not aware of any systematic bias such that only highly symptomatic clients would have been selected for monitoring.

\section{Compared groups, hypotheses and statistics}

The following criteria were applied to inform the choice of groups to be compared with the RCT group:

1. The DRI/HADS data for pain-patients had been collected prior to treatment.
2. The comparison groups came from

Scandinavia so as to make it plausible that general societal conditions were approximately comparable with those of the RCT patients residing in Denmark.

3. A sufficient sample size, at least approximating the largest RCT-patient group.

4. The sample should include one of the more common categories of chronic pain.

\section{The Swedish National Register for Pain} Rehabilitation (NRS) contains pre-treatment DRI and HADS-data fulfilling the criteria and was chosen as a comparison group. The NRS is a national documentation system in which patient data from the vast majority of all providers of rehabilitation programs for chronic pain in Sweden are collected. The data are published in annual reports. For the DRI comparison, the NRS annual report $2008,{ }^{13}$ which is the last one providing DRI data, was chosen. For the HADS-comparison, the most recently available NRS data, published in the annual report 2013 was chosen. ${ }^{14}$ The NRS annual report 2008 provides DRI data prior to eventual treatment for three subgroups: (1) patients who had only been assessed (no treatment offered); (2) patients who participated in a rehabilitation program without the goal of increasing working ability; and, (3) patients who participated in a rehabilitation program with the intended outcome of improving working ability. The DRI score of the RCT sample was compared with the DRI scores of these three NRS subgroups. The hypothesis was that RCT patients suffer from painrelated disability that is comparable to or higher than that of the Swedish pain patients (alternative hypothesis, $\mathrm{H} 1$ ), i.e. the DRI score of the RCT-patients is of the same size - or higher than - the score of the painpatients. The null hypothesis $(\mathrm{H} 0)$ is that the RCT patients do not suffer from pain-related 
disability to a degree that is comparable to that of the Swedish pain patients i.e. the DRI-score of the RCT-patients is lower than that of the pain-patients.

For HADS, the NRS annual report 2013 provides data for all patients measured prior to eventual treatment (i.e. without the differentiation of subgroups described for the DRI data in the 2008 annual report), and the HADS scores for anxiety and depression of the RCT sample are compared with the scores for this overall NRS sample. The alternative hypothesis (H1) was that RCT patients show a higher degree of anxiety and depression than the pain patients. The hypothesis is based on the assumption that RCT patients are more anxious and depressed as a consequence of severe traumatization and other factors that are (typically) not present for pain-patients. The null hypothesis (H0) is that the HADS-scores of the RCT-patients are comparable to, or lower than, those of the pain-patients.

The development of the DRI score is based on the comparison of groups with an a priori hierarchy of increasing degrees of pain-related disability. The DRI-validation study provides data for degrees of disability ranging from zero/very low (healthy persons) to extreme (wheelchair bound multiple sclerosis patients) and in-between (with ascending degrees of disability) for groups suffering from pain located in/caused by: (1) neck/shoulder, (2) neck/shoulder plus low back pain, (3) arthritis of the knee and (4) arthritis of the hip (groups (3) and (4) waiting for implant surgery). Comparing the DRI scores of the RCT patients with the scores for these subgroups, ordered in a hierarchy of severity of pain-related dysfunction, provides qualitative information about where the degree of dysfunction of the RCT patients is approximately located. In order to achieve such a qualitative comparison, i.e. to find out whether RCT-clients score closer to the lower or higher end of the functional disability, the RCT DRI scores were also compared with the data provided in the DRI-validation study. ${ }^{6}$ This comparison is explorative and no a priori hypotheses are formulated (besides the implicit assumption that the DRI score of the RCT-patients is higher than that of the healthy subjects). Since the validation study does not provide means (but medians) and standard deviations, no parametric comparison was possible. Instead, $\mathrm{Chi}^{2}$ tests were computed in order to test the (non)equivalence with the pain-subgroups: the number of cases in the RCT sample with a score lower/greater than the median of the comparison group were compared with the number of cases below $(50 \%)$ and above $(50 \%)$ the median in the comparison group. In groups with an uneven size, the extra case was added to the above median subgroup, for example, in the case of 65 subjects, the above median group was found to be 33 and the sub-median group 32 .

To investigate the presumptive underestimation of psychiatric disability of the refugee-sample in the Palic et al. study, ${ }^{5}$ average effect sizes for the differences between the refugee and the psychiatric samples were computed. The hypothesis is based on the logic that exclusion of patients with psychosis and substance abuse cause artificially low scores in the items measuring these problems. The comparison with psychiatric patients actually suffering from these problems is accordingly biased. The computation of effect sizes included in the first step all 12 HoNOS items. In a second step, only 11 items were included, omitting the psychotic symptoms item. In a third step, 11 items were included, omitting the substance abuse item. In the fourth step, both critical items were omitted, leaving 10 
items included in the effect size computation. The hypothesis of underestimation of the psychiatric disability of the refugees is in this way, at least tentatively, testable: $\mathrm{H} 1$ that exclusion of the critical items increases the effect sizes and $\mathrm{H} 0$ that there is no increase (or decrease) of effect sizes.

Pearson correlations were computed using SPSS version $19 .{ }^{9}$ Student t-tests ${ }^{10}$ were applied for parametric group comparisons and $\mathrm{chi}^{2}$-tests ${ }^{11}$ for categorical/ordinal data. With effect size computations, ${ }^{12}$ correction for sample size was applied. The average effect sizes for the difference between the CPTP refugee sample and psychiatric inpatients were calculated manually, based on the item-wise effect sizes provided by Palic et al. ${ }^{5}$ As far as was relevant, two- sided hypothesis testing, the assumption of unequal variances and an alpha level of $\leq 0.01$ as criterion for significance were applied.

Descriptive information is provided for all available RCT cases in Table 1 below
("Total Sample") and for all cases with available DRI ("DRI Sample") and HADS ("HADS Sample") data. The description partly follows the categories used by Palic et. al. ${ }^{5}$ and includes information from this study.

\section{Results and conclusions}

The available data indicate that $77 \%$ of all RCT patients came from four countries of origin: Iraq (47.9\%), Afghanistan $(12.8 \%)$, Iran (9.5\%) and Lebanon (7\%), and have either Arabic (59\%) or Farsi $(17.7 \%)$ as their primary language. $63 \%$ of the refugees in the CPTP sample were in need of an interpreter (no information is provided regarding any differences between those with and without the need of interpretation). Further descriptive information is given in Table 1.

In order to assess whether gender and age could lead to bias in the study for the DRI and HADS anxiety and depression scores, t-tests comparing male and female patients and Pearson-correlations with age

Table 1: Descriptive data for the RCT DRI, HADS and total sample (either DRI or HADS data available) and the Clinic for PTSD and Transcultural Psychiatry, Aarhus University Hospital (CPTP)-refugee sample. ${ }^{5}$

\begin{tabular}{|c|c|c|c|c|}
\hline & $\begin{array}{l}\text { RCT - DRI } \\
\text { Sample }\end{array}$ & $\begin{array}{l}\text { RCT - HADS } \\
\text { Sample }\end{array}$ & $\begin{array}{l}\text { RCT - Total } \\
\text { Sample }\end{array}$ & CPTP Sample \\
\hline $\mathrm{n}=$ & 197 & 147 & 259 & 448 \\
\hline $\begin{array}{l}\text { Mean age } \\
\text { Sd } \\
\text { Range }\end{array}$ & $\begin{array}{l}44.69 \\
(7.71) \\
16-64\end{array}$ & $\begin{array}{l}43.87 \\
(8.21) \\
16-64\end{array}$ & $\begin{array}{l}44.11 \\
(7.92) \\
16-64\end{array}$ & $\begin{array}{l}40.5 \\
(8.57) \\
20-61\end{array}$ \\
\hline$\%$ female & 34 & 35.4 & 34.7 & 45.5 \\
\hline$\%$ Country of origin, $\mathrm{n}=$ & 185 & 140 & 243 & 448 \\
\hline Middle East* & 80.5 & 89.3 & 83.1 & 65.0 \\
\hline Balkans & 5.4 & 4.3 & 5.4 & 25.9 \\
\hline Other & 14.1 & 6.4 & 11.6 & 8.7 \\
\hline$\%$ Primary Language, $\mathrm{n}=$ & 190 & 141 & 248 & \\
\hline Arabic & 53.7 & 66.0 & 59.0 & \\
\hline
\end{tabular}

Data collection and available RCT samples 
were calculated. There are no differences with respect to gender on the HADS-scales (both $\mathrm{p}>.90$ ) and no significant (both $\mathrm{p}>$ $.74)$ or substantial correlations with age (-.01 for anxiety and .03 for depression). Female RCT patients have mean $(61.87, \mathrm{Sd}=$ 17.38) DRI-scores that are almost significantly higher $(\mathrm{t}=2.40, \mathrm{p}=.018)$ than that of men (54.77, $\mathrm{Sd}=23.60)$. There is a small $(\mathrm{r}=.24)$ but significant $(\mathrm{p}<.01)$ correlation between age and DRI score. It is concluded that gender and age have no influence on HADS scores. However, these factors could be potential confounders for DRI-scores since female gender and higher age are associated with higher DRI-scores.

The results for the RCT-DRI comparison with the Swedish NRS samples and the medians for the pain subgroups from the DRI validation study are given in Table 2 . The mean of the RCT-sample is larger than the mean of the three NRS groups and the difference is significant for the subgroup receiving rehabilitation without the goal of improving working ability. The healthy subjects ( $n=943$, median age 43 , female $48.1 \%$ ) described in the DRI-validation study have a negligible DRI median score of 0.8 and the proportion of RCT-patients exceeding this score $(98.5 \%)$ is accordingly highly significant $\left(\mathrm{chi}^{2}=157.31, \mathrm{p}<.01\right)$. The proportion of RCT- patients with DRI scores greater $(6.1 \%)$ than the median for multiple sclerosis patients is significantly lower. The proportions above the medians for arthritis of the hip and knee patients $(42.1 \%$ and $65.5 \%$ ) are not significantly different but the proportions are significantly higher for the RCT sample compared to the patients with neck/shoulder with and without additional low back pain $(80.7 \%$ and $88.8 \%$ respectively).

Table 2: Median, means and standard deviations (Sd) for Disability Rating Index (DRI) scores (mean of 12 items) for the RCT-DRI sample and scores reported in the Swedish annual report 2008 of the National Register for Pain Rehabilitation (NRS).

\begin{tabular}{|c|c|c|c|c|c|c|c|c|c|}
\hline & \multirow{2}{*}{$\begin{array}{l}\text { RCT } \\
(n=197)\end{array}$} & \multicolumn{3}{|c|}{ NRS annual report 2008} & \multicolumn{5}{|c|}{ DRI evaluation study } \\
\hline & & $\begin{array}{l}\begin{array}{l}\text { NRS } 1 \\
(\mathrm{n}= \\
1470)\end{array}\end{array}$ & $\begin{array}{l}\text { NRS 2 } \\
n=186)\end{array}$ & $\begin{array}{l}\text { NRS } 3 \\
(\mathrm{n}=818)\end{array}$ & $\begin{array}{l}\text { Neck/ } \\
\text { shoulder } \\
(n=127)\end{array}$ & $\begin{array}{l}\text { Neck/ } \\
\text { Sh. + } \\
\text { LBP } \\
(n=39)\end{array}$ & $\begin{array}{l}\text { Arthritis } \\
\text { knee } \\
(\mathrm{n}=24)\end{array}$ & $\begin{array}{l}\text { Arthritis } \\
\text { hip } \\
(n=23)\end{array}$ & $\begin{array}{l}\text { Multiple } \\
\text { sclerosis } \\
(n=16)\end{array}$ \\
\hline Median & 62 & & & & 27 & 39 & 50 & 65 & 85 \\
\hline Mean & 57 & 54 & 50 & 53 & & & & & \\
\hline $\mathrm{Sd}$ & 22 & 21 & 20 & 18 & & & & & \\
\hline$t=$ & & 1.92 & 3.35 & 2.48 & & & & & \\
\hline $\operatorname{chi}^{2}=$ & & & & & 58.96 & 15.39 & 2.22 & .85 & 33.53 \\
\hline $\mathrm{p}$ & & .056 & .001 & .014 & $<.01$ & $<.01$ & $=.14$ & $=.36$ & $<.01$ \\
\hline $\mathrm{d}=$ & & .15 & .34 & .22 & & & & & \\
\hline
\end{tabular}

NRS 1 - patients who have only been assessed

NRS 2 - patients who have participated in rehabilitation without ambition to improve working ability (t)/(d)/(chi) NRS 3 - patients with the ambition to improve working ability

(d) Results for Student t-test comparisons and Cohen effect sizes and $\mathrm{chi}^{2}$ results for comparisons of the proportion of RCT cases $>$ the median of each pain-subsample from the DRI validation study. ${ }^{4}$ 
Table 3: Medians, means and standard deviations (Sd) for Hospital Anxiety and Depression Scale (HADS) sum-scores for anxiety and depression for the RCT refugee sample and pain patients reported in the annual report 2013 of the National Register for Pain Rehabilitation (NRS).

\begin{tabular}{l|ll|ll}
\hline & \multicolumn{2}{|c|}{ RCT } & \multicolumn{2}{c}{ NRS annual report 2013 } \\
\hline & HADS- & HADS- & HADS- & HADS- \\
& Anxiety & Depression & Anxiety & Depression \\
$\mathrm{n}=$ & 147 & 147 & 1994 & 1996 \\
Median & 18 & 16 & 9 & 8 \\
Mean & 16,48 & 15.50 & 8.9 & 8.3 \\
$\mathrm{Sd}$ & 4.29 & 4.15 & 4.86 & 4.46 \\
$\mathrm{t}=$ & & 20.48 & 20.19 \\
$\mathrm{p}<$ & & & .000 & .000 \\
$\mathrm{~d}=$ & & & 1.57 & 1.62 \\
\hline
\end{tabular}

Results for Student t-test comparisons and Cohen effect sizes (d).

Meanwhile, the three NRS-groups are comparable in age $($ mean $=43 / 41 / 40, \mathrm{Sd}=$ $10 / 10 / 9)$. The proportion of females $(68 / 71 / 79 \%)$ is much higher than in the RCT-sample (34\%). Also for all pain subgroups described in the validation study, the proportion of female patients is greater $(>50 \%)$ than in the RCT-DRI sample. The age-median for the RCT sample is 44 and for the pain-subgroups in the validation studies $42 / 39 / 73 / 70 / 46$; i.e. the RCT age-median is not dramatically higher than the lowest medians in the pain-subgroups. Given a higher proportion of females in all comparison groups and no comparison group being dramatically younger than the RCT- sample, it is not probable that the high scores in the RCT sample are attributable to confounding by sex and age.

It is concluded that $\mathrm{HO}$ has to be rejected, i.e. RCT-patients have an at least equal degree of functional disability as Swedish pain-patients. The DRI-scores in comparison with the pain subgroups in the DRI-validation study are to be found in the upper end of the hierarchy, only exceeded by wheelchair bound multiple sclerosis patients.

The results for the HADS-comparisons are presented in Table 3. Both the anxiety and depression mean scores of the RCT sample are much larger than those of the Swedish pain patients. The comparisons are statistically significant and the effect sizes are very large. It is concluded that $\mathrm{HO}$ has to be rejected; the degree of anxiety and depression is much higher than what is seen in Swedish pain patients.

The results for the re-analyses of the effect sizes on differences between the CPTP refugee sample and psychiatric in-patients is given in Table 4. All averaged effect sizes increase when either the substance abuse or the hallucination/delusion item is excluded. With both items excluded, the averaged effect size for the six refugee psychiatric subgroup comparisons increases from .33 to .54 , i.e. by $64 \%$. The higher degree of disability of the refugee-sample is small $(\mathrm{d}=$ .15) relative to the dementia-group. All other differences are substantial, varying between medium (.50, addiction group) to 
Table 4: Health of Nation Outcome Scales (HoNos) sum score (12 items) for refugee patients at intake for rehabilitation at the Clinic for PTSD and Transcultural Psychiatry, Aarhus University Hospital (CPTP) and psychiatric in-patients at intake at the Psychiatric Center North Zealand. Group wise mean effect sizes (d) of differences between the refugee and all other groups for all items and for the substance abuse and hallucination items and both excluded; the effect sizes are computed based on the item-wise effect sizes provided by Palic et al. ${ }^{5}$

\begin{tabular}{|c|c|c|c|c|c|c|c|}
\hline & $\begin{array}{l}\text { СPTP } \\
\text { refugees }\end{array}$ & $\begin{array}{l}\text { Schizo- } \\
\text { phrenia }\end{array}$ & $\begin{array}{l}\text { Affective } \\
\text { disorders }\end{array}$ & $\begin{array}{l}\text { Anxiety } \\
\text { disorders }\end{array}$ & $\begin{array}{l}\text { Personal. } \\
\text { disorders }\end{array}$ & Addiction & Dementia \\
\hline $\mathrm{n}=$ & 448 & 3175 & 3081 & 1781 & 1030 & 950 & 950 \\
\hline $\begin{array}{l}\text { Sum for all items } \\
\text { (Sd) }\end{array}$ & $\begin{array}{l}15.70 \\
(5.60)\end{array}$ & $\begin{array}{l}12.16 \\
(5.87)\end{array}$ & $\begin{array}{l}9.90 \\
(5.19)\end{array}$ & $\begin{array}{l}9.01 \\
(4.96)\end{array}$ & $\begin{array}{l}10.24 \\
(5.17)\end{array}$ & $\begin{array}{l}13.32 \\
(6.54)\end{array}$ & $\begin{array}{l}15.55 \\
(7.32)\end{array}$ \\
\hline $\begin{array}{l}\text { Sum without } \\
\text { substance and } \\
\text { hallucination item }\end{array}$ & 14.95 & 9.43 & 9.34 & 8.14 & 8.93 & 9.86 & 13.83 \\
\hline \multicolumn{2}{|l|}{ Mean $d$ all items } & .32 & .49 & .62 & .51 & .17 & .05 \\
\hline \multicolumn{2}{|c|}{$\begin{array}{l}\text { Mean } d \text { without substance } \\
\text { abuse item }\end{array}$} & .40 & .57 & .71 & .61 & .44 & .08 \\
\hline \multicolumn{2}{|c|}{$\begin{array}{l}\text { Mean d without hallucination } \\
\text { item }\end{array}$} & .46 & .52 & .67 & .55 & .20 & .11 \\
\hline \multicolumn{2}{|c|}{$\begin{array}{l}\text { Mean } d \text { without substance } \\
\text { abuse and hallucination items }\end{array}$} & .56 & .61 & .77 & .67 & .50 & .15 \\
\hline
\end{tabular}

large (.77, anxiety disorder group) effect sizes.

It is concluded that the alternative hypothesis $(\mathrm{H} 1)$ is supported: the exclusion of patients with substance abuse and psychotic symptoms from the refugee-sample led to the Palic et al. ${ }^{5}$ study underestimating the extent of disability within the refugee patients, relative to the psychiatric inpatient groups. Controlling for the exclusion-bias reinforces the conclusion that refugee patients have a considerably higher degree of psychiatric disability than psychiatric inpatients.

In summary, it can be concluded that RCT patients show a functional disability that is at least on a par with inpatients suffering from chronic pain. The degree of anxiety and depression is much higher than the levels found in chronic pain patients. The refugee-patients in the study of Palic et al. ${ }^{5}$ have a higher total disability than the psychiatric inpatients included in the study.

\section{Discussion}

An obvious limitation of the presented and compared DRI and HADS data from RCT patients is that these stem from only $44 \%$ all patients receiving treatment. However, the findings and conclusions restricted to this subgroup of refugees would be large enough to remain relevant even when assuming the most extreme selection bias -that all not included patients would have shown much lower symptom-scores.

A possible opposite objection is that the presented data might be representative for RCT-clients but that these constitute only a very small, highly selected refugee-subgroup with an extreme symptom load. As mentioned previously, patients accepted into the 
RCT program are typically more problematic and judged to be in need of multidisciplinary rehabilitation, as compared to patients treated at other Danish centers. It can, however, be stated that this group is not negligibly small, since about 100 cases per year receive rehabilitation treatment at RCT. Also, a considerable proportion of the patients receiving treatment at other Danish centers can be assumed to belong to the severely symptomatic subgroup. For example, $31.25 \%$ of the CPTP sample were torture survivors. $^{5}$

A third potential objection is that the RCT data may be affected by patients purposefully exaggerating their complaints for secondary gains, such as, to continue receiving social security benefits. Here it is important to note that the observer-rated HoNOS results showed high degrees of symptomatology and that the RCT group as a whole does not express as high DRI-scores as found among patients suffering from multiple sclerosis. Such arguments do not, however, exclude the objection that a typical refugee patient might purposefully exaggerate his own degree of disability, but not to a degree that would risk credibility. The same objection however could also be made for a majority of pain patients, since unambiguous objective somatic findings underlying chronic pain are only rarely found. Among the pain patients mentioned in this paper, the wheelchair bound multiple sclerosis and a proportion of the arthritis patients (those with pronounced X-ray findings) would probably be the only patients having a lowered risk for objections regarding credibility.

The available comparative data support the experience of most clinicians that traumatized refugee patients in Denmark suffer from multiple disabling complaints, including pain and emotional distress, with high degrees of symptom-load. The question, which cannot be answered at this time, is whether a single complaint, such as PTSD, can explain the high levels of disability, anxiety, and depression seen in these patients. It is the feeling of experienced clinicians working with torture survivors, that their symptoms are multifactorial. As mentioned in the Istanbul Protocol, ${ }^{15}$ it is important to explore the possibilities of other conditions related to the torture experience, such as traumatic brain injury, chronic pain, sleep disturbance, secondary substance abuse, and metabolic syndrome resulting from prolonged stress exposure (hypertension, diabetes).

Acknowledging the multifactorial nature of torture survivors' symptoms, we must ask ourselves whether the evaluation and treatment strategies currently practiced are adequate to address the multiple barriers that these patients must overcome to regain a functional, productive, and satisfying life. 
References

1. Patel, N, Kellezi, B, Williams ACDC. Psychological, social and welfare interventions for psychological health and well-being of torture survivors (Review). Cochrane Database of Systematic Reviews 2014, Issue 11. ART. No.: CD009317. DOI: 10.1002/14651858.CD009317.pub.

2. Slobodin O, De Jong J. Mental health interventions for traumatized asylum seekers and refugees: What do we know about their efficacy? Int J Soc Psychiatry. 2015 Feb;61(1):17-26.

3. Fazel M, Wheeler J, Danesh J. Prevalence of serious mental disorder in 7000 refugees resettled in western countries: a systematic review. Lancet. 2005 Apr 9-15;365(9467):1309-14.

4. Panha P, Sopheap T, Harlacher U, Polatin P. Education as Treatment for Chronic Pain in Survivors of Torture and Other Violent Events in Cambodia: Experiences with Implementation of a Group-Based "Pain School" and Evaluation of Its Effect in a Pilot Study. Journal of Applied Biobehavioral Research, 2014 Mar;19(1)53-69.

5. Palic S, Lind Kappel M, Stougaard Nielsen $M$, Carlsson J, Bech P. Comparison of psychiatric disability on the health of nation outcome scales (HoNOS) in resettled traumatized refugee outpatients and Danish inpatients. BMC Psychiatry. 2014 Dec 18;14:330.

6. Salén, BA, Spangfort, EV, Nygren, AL, Nordemar R). The Disability Rating Index: an instrument for the assessment of disability in clinical settings. J Clin Epidemiol. 1994 Dec;47(12):1423-35.

7. Hawker GA, Mian S, Kendzerska T, French M. Measures of adult pain. Arthritis Care Res 63 (S11), S240-S252.

8. Zigmond AS, Snaith RP, "The hospital anxiety and depression scale". Acta Psychiatr Scand. 1983 Jun;67(6):361-370.

9. IBM Corp. Released 2015. IBM SPSS Statistics for Windows, Version 23.0. Armonk, NY: IBM Corp.

10. In-silico. T-test statistical calculator [Internet]. 2016-2016. [accessed 2015 Mar 1]. Available from: http://in- silico.nettools/statististics/ttest.

11. Stangroom J. Chi-square calculator [Internet]. 2016. [accessed 2015 Mar 1]. Available from: www.socscistatistics.com/tests/chisquare.

12. Psychometrica Institut für Psychologische Diagnostik. Berechnung von Effekstärken (2) Mittelwertsunterschiede zweier verscheiden grosser Gruppen (Cohen's d, Hedges' g). [Internet]. 2014-2016 [cited 2015 Mar 1]. Available from: www.psychometrica.de/effekstaerke.html.

13. Nationella Registret Över Smärtrehabilitering.
Årsrapport 2008. [posted 2009 Jan 9; accessed 2015 Mar 1]. Available from: www.ucr.uu.se/nrs/ index.php/arsrapporter/cat view/55-arsrapporter.

14. Nationella Registret Över Smärtrehabilitering. Rapport 2014:1.-Årsrapport 2013 del 1. [updated 2014 Aug 9; accessed 2015 Mar 1]. Available from: www.ucr.uu.se/nrs/index.php/arsrapporter/cat view/55-arsrapporter.

15. UN Office of the High Commissioner for $\mathrm{Hu}-$ man Rights (OHCHR), Manual on the Effective Investigation and Documentation of Torture and Other Cruel, Inhuman or Degrading Treatment or Punishment ("Istanbul Protocol"), 2004, HR/P/PT/8/Rev.1 Available at: http://www.refworld.org/docid/4638aca62.html 